\title{
Multiple periodic solutions for resonant difference equations
}

\author{
Jianming Zhang, Shuli Wang ${ }^{*}$, Jinsheng Liu and Yurong Cheng
}

"Correspondence:

zwangshuli@163.com

College of Mathematics, Taiyuan

University of Technology, Taiyuan,

Shanxi 030024, P.R. China

\begin{abstract}
In this paper, we study the existence of multiple periodic solutions for nonlinear second-order difference equations with resonance at origin. The approach is based on critical point theory, minimax methods, homological linking and Morse theory.
\end{abstract}

Keywords: difference equations; periodic solutions; homological linking; critical group; Morse theory

\section{Introduction}

In this paper, we consider the existence of multiple periodic solutions for the following nonlinear difference equations:

$$
\text { (P) }\left\{\begin{array}{l}
-\triangle^{2} u(k-1)=\lambda_{m} u(k)+f(k, u(k)), \quad k \in \mathbb{Z}[1, N] \\
u(0)=u(N), \quad u(1)=u(N+1),
\end{array}\right.
$$

where $N>3$ is a fixed integer, $\Delta u(k)=u(k+1)-u(k), \Delta^{2} u(k)=\Delta(\Delta u(k)), f(k, \cdot): \mathbb{R} \rightarrow \mathbb{R}$ is a differential function satisfying

$$
f(k, 0)=0, \quad k \in \mathbb{Z}[1, N]
$$

and $\lambda_{m}$ is the $m+1$ th eigenvalue of the linear periodic boundary value problem

$$
\left(P_{0}\right) \quad\left\{\begin{array}{l}
-\triangle^{2} u(k-1)=\mu u(k), \quad k \in \mathbb{Z}[1, N], \\
u(0)=u(N), \quad u(1)=u(N+1) .
\end{array}\right.
$$

Since (1.1) implies that $(P)$ possesses a trivial periodic solution $u \equiv 0$, we are interested in finding nontrivial periodic solutions for $(P)$. It follows from [1] that all the eigenvalues of $\left(P_{0}\right)$ are $\mu_{k}=4 \sin ^{2} \frac{k \pi}{N}, k \in \mathbb{Z}[0, N-1]$. Thus $\mu_{0}=0, \mu_{j}=\mu_{N-j}$ for $j \in \mathbb{Z}\left[1, N_{1}\right]$, where

$$
N_{1}= \begin{cases}\frac{N-1}{2} & \text { if } N \text { is odd } \\ \frac{N}{2} & \text { if } N \text { is even. }\end{cases}
$$

For the convenience of later use, we denote by $0=\lambda_{0}<\lambda_{1}<\cdots<\lambda_{N_{1}}$ the distinct eigenvalues of $\left(P_{0}\right)$. Moreover, if $N$ is odd then all eigenvalues of $\left(P_{0}\right)$ are multiplicity two except $\lambda_{0}$, 
and let

$\phi_{0}, \phi_{1}, \psi_{1}, \ldots, \phi_{N_{1}}, \psi_{N_{1}}$

be the corresponding orthonormal eigenvectors; if $N$ is even then all eigenvalues are multiplicity two except $\lambda_{0}$ and $\lambda_{N_{1}}$, and let

$\phi_{0}, \phi_{1}, \psi_{1}, \ldots, \phi_{N_{1}-1}, \psi_{N_{1}-1}, \phi_{N_{1}}$

be the corresponding orthonormal eigenvectors.

Now we establish the variational framework associated with $(P)$. Set

$$
u=(u(1), u(2), \ldots, u(N))^{T}, \quad \mathbf{f}(u)=(f(1, u(1)), f(2, u(2)), \ldots, f(N, u(N)))^{T}
$$

and

$$
A=\left(\begin{array}{ccccccc}
2 & -1 & 0 & \cdots & 0 & 0 & -1 \\
-1 & 2 & -1 & \cdots & 0 & 0 & 0 \\
\vdots & \vdots & \vdots & & \vdots & \vdots & \vdots \\
0 & 0 & 0 & \cdots & -1 & 2 & -1 \\
-1 & 0 & 0 & \cdots & 0 & -1 & 2
\end{array}\right)_{N \times N} .
$$

Then we can rewrite $(P)$ and $\left(P_{0}\right)$ as

$$
A u=\lambda_{m} u+\mathbf{f}(u) \text { and } A u=\mu u,
$$

respectively.

Let $E=\mathbb{R}^{N}$ with inner product $\langle u, v\rangle=\sum_{k=1}^{N} u(k) v(k)$ and norm $\|u\|=\sqrt{\langle u, u\rangle}$. Then

$$
0=\lambda_{0}\|u\|^{2} \leq\langle A u, u\rangle \leq \lambda_{N_{1}}\|u\|^{2}, \quad u \in E .
$$

For $p \geq 1$, define $\|u\|_{p}=\left(\sum_{k=1}^{N}|u(k)|^{p}\right)^{1 / p}$, then there exist positive numbers $a_{p}, b_{p}$ such that

$$
a_{p}\|u\| \leq\|u\|_{p} \leq b_{p}\|u\|, \quad \forall u \in E .
$$

Define the functional $J: E \rightarrow \mathbb{R}$ by

$$
J(u)=\frac{1}{2}\langle A u, u\rangle-\frac{1}{2} \lambda_{m}\|u\|^{2}-\sum_{k=1}^{N} F(k, u(k)), \quad u \in E,
$$

where $F(k, x)=\int_{0}^{x} f(k, s) d s$. Then $J \in C^{2}(E, \mathbb{R})$ with derivatives

$$
\begin{aligned}
& J^{\prime}(u)=A u-\lambda_{m} u-\mathbf{f}(u), \quad u \in E, \\
& J^{\prime \prime}(u)=A-\lambda_{m} I_{N}-\mathbf{f}^{\prime}(u), \quad u \in E,
\end{aligned}
$$


where $I_{N}$ is the identity matrix of order $N, \mathbf{f}^{\prime}(u)=\operatorname{diag}\left\{f^{\prime}(1, u(1)), \ldots, f^{\prime}(N, u(N))\right\}$. Hence the periodic solutions of $(P)$ are exactly the critical points of $J$ or $-J$ in $E$.

We assume that the nonlinearity $f$ satisfies the following conditions:

$\left(f_{0}\right) \quad f^{\prime}(k, 0)=0$ for $k \in \mathbb{Z}[1, N]$.

$\left(f_{0}^{+}\right)$There exists $\delta>0$ such that

$$
F(k, x) \geq 0, \quad k \in \mathbb{Z}[1, N],|x| \leq \delta .
$$

$\left(f_{0}^{-}\right)$There exists $\delta>0$ such that

$$
F(k, x) \leq 0, \quad k \in \mathbb{Z}[1, N],|x| \leq \delta .
$$

$\left(f_{\infty}^{+}\right)$There exist $r>0$ and $\theta>2$ such that

$$
0 \leq \theta F(k, x) \leq f(k, x) x, \quad k \in \mathbb{Z}[1, N],|x|>r .
$$

$\left(f_{\infty}^{-}\right)$There exist $r>0$ and $\theta>2$ such that

$$
0 \geq \theta F(k, x) \geq f(k, x) x, \quad k \in \mathbb{Z}[1, N],|x|>r .
$$

(f) For any a fixed number $\tau>\theta$, there exists $C>0$ such that

$$
|F(k, x)| \leq C\left(1+|x|^{\tau}\right), \quad k \in \mathbb{Z}[1, N], x \in \mathbb{R} .
$$

Therefore we regard the problem $(P)$ as resonance at origin under the assumption $\left(f_{0}\right)$.

Critical point theory has been widely used to study the existence of periodic solutions and solutions for nonlinear difference boundary value problems since the first result was established by using variational methods in 2003 (see [2]). Since then, by using critical point theory, minimax methods and Mores theory, the existence of solutions for nonresonant difference equations has been extensively investigated (see [3-9] and the references therein). As for resonant cases, Zhu and Yu [10] applied critical point theory to study the existence of positive solutions for a second order nonlinear discrete Dirichlet boundary value problem

$$
\left\{\begin{array}{l}
-\triangle^{2} u(k-1)=f(k, u(k)), \quad k \in \mathbb{Z}[1, N], \\
u(0)=0, \quad u(N+1)=0
\end{array}\right.
$$

when nonlinearity $f$ is odd and resonant at infinity. Zheng and Xiao [11] employed critical groups and the mountain pass theorem to study the existence of nontrivial solutions for (1.7) when the nonlinearity $f(k, u)=V^{\prime}(u)$ and is resonant at infinity. Liu et al. [12] used Morse theory, critical point theory and minimax methods to study the existence of multiple solutions for (1.7) with resonance at both infinity and origin, one can refer to [13, 14].

However, we note that only a few papers concern the existence of periodic solutions for difference equations with resonance. In 2011, Zhang and Wang [15] used variational 
methods and Morse theory to study the multiplicity of periodic solutions for $(P)$ with double resonance between two consecutive eigenvalues at infinity. The main aim of this paper is to study the multiplicity of nonzero periodic solutions for $(P)$ with resonance at origin. The approach is based on critical theory, Morse theory and homological linking.

The rest of this paper is organized as follows. In Section 2, we collect some useful preliminary results about Morse theory. In Section 3, we give some auxiliary results. Our main results and proofs will be given in Section 4.

\section{Preliminaries about Morse theory}

In this section, we recall some facts about Morse theory and critical groups [16, 17]. Let $E$ be a real Hilbert space. We say that $J$ satisfies the (PS) condition if every sequence $\left\{u_{n}\right\} \subset E$ such that $J\left(u_{n}\right)$ is bounded and $J^{\prime}\left(u_{n}\right) \rightarrow 0$ as $n \rightarrow \infty$ has a convergent subsequence.

Suppose that $J \in C^{1}(E, \mathbb{R})$ is a functional satisfying the (PS) condition. Let $u_{0}$ be an isolated critical point of $J$ with $J\left(u_{0}\right)=c \in \mathbb{R}$, and let $U$ be a neighborhood of $u_{0}$. The group

$$
C_{q}\left(J, u_{0}\right):=H_{q}\left(J^{c} \cap U,\left(J^{c} \backslash\left\{u_{0}\right\}\right) \cap U\right), \quad q \in \mathbb{Z}
$$

is called the $q$ th critical group of $J$ at $u_{0}$, where $J^{c}=\{u \in E \mid J(u) \leq c\}, H_{*}(A, B)$ denotes the singular relative homology group of the topological pair $(A, B)$ with coefficient field $\mathbb{F}$. Define

$$
\mathcal{K}=\left\{u \in E \mid J^{\prime}(u)=0\right\} .
$$

Assume that $\mathcal{K}$ is a finite number. Take $a<\inf J(\mathcal{K})$. The group

$$
C_{q}(J, \infty):=H_{q}\left(E, J^{a}\right), \quad q \in \mathbb{Z}
$$

is called the $q$ th critical group of $J$ at infinity (see [18]). The Morse type numbers of the pair $\left(E, J^{a}\right)$ are defined by $M_{q}:=\sum_{u \in \mathcal{K}} \operatorname{dim} C_{q}(J, u)$. Denote by $\beta_{q}:=\operatorname{dim} C_{q}(J, \infty)$ the Betti numbers of the pair $\left(E, J^{a}\right)$. By Morse theory, the relationship between $M_{q}$ and $\beta_{q}$ is described by

$$
\sum_{j=0}^{q}(-1)^{q-j} M_{j} \geq \sum_{j=0}^{q}(-1)^{q-j} \beta_{j}
$$

and

$$
\sum_{q=0}^{\infty}(-1)^{q} M_{q}=\sum_{q=0}^{\infty}(-1)^{q} \beta_{q} .
$$

From $M_{q} \geq \beta_{q}$, for each $q \in \mathbb{N}$, it follows that if $C_{l}(J, \infty) \nsucceq 0$ for some $l \in \mathbb{N}$, then $J$ must have a critical point $u^{*}$ with $C_{l}\left(J, u^{*}\right) \varsubsetneqq 0$. If $\mathcal{K}=\left\{u^{*}\right\}$, then $C_{q}(J, \infty) \cong C_{q}\left(J, u^{*}\right)$ for all $q \in \mathbb{N}$. Thus if $C_{l}(J, \infty) \nsucceq C_{l}\left(J, u^{*}\right)$ for some $l \in \mathbb{N}$, then $J$ must have a new critical point.

For some $k \in \mathbb{Z}$, define

$$
\delta_{q, k} \mathbb{F}:= \begin{cases}\mathbb{F}, & q=k, \\ 0, & q \neq k .\end{cases}
$$


Then

$$
C_{q}\left(J, u_{0}\right) \cong \begin{cases}\delta_{q, 0} \mathbb{F} & \text { if } u_{0} \text { is an isolated local minimum of } J, \\ \delta_{q, l} \mathbb{F} & \text { if } u_{0} \text { is an isolated local maximum of } J \text { and } l=\operatorname{dim} E<\infty\end{cases}
$$

Suppose that $J \in C^{2}(E, \mathbb{R})$ and $u_{0} \in \mathcal{K}$. Then $J^{\prime \prime}\left(u_{0}\right)$ is a self-adjoint linear operator on $E$. The dimension of the largest negative space of $J^{\prime \prime}\left(u_{0}\right)$ is called the Morse index of $J$ at $u_{0}$, and the dimension of the kernel of $J^{\prime \prime}\left(u_{0}\right)$ is called the nullity of $J$ at $u_{0}$. We say that $u_{0}$ is nondegenerate if the nullity of $J$ at $u_{0}$ is zero, i.e., $J^{\prime \prime}\left(u_{0}\right)$ has a bounded inverse. For an isolated critical point, the following important result is valid.

Proposition 2.1 ([16, 17]) Suppose that $u_{0}$ is an isolated critical point of $J \in C^{2}(E, \mathbb{R})$ with finite Morse index $\mu\left(u_{0}\right)$ and nullity $v\left(u_{0}\right)$.

(i) $C_{q}\left(J, u_{0}\right) \cong 0$ for $q \notin\left[\mu\left(u_{0}\right), \mu\left(u_{0}\right)+v\left(u_{0}\right)\right]$.

(ii) If $u_{0}$ is nondegenerate, then $C_{q}\left(J, u_{0}\right) \cong \delta_{q, \mu\left(u_{0}\right)} \mathbb{F}$.

(iii) If $C_{l}\left(J, u_{0}\right) \supsetneqq 0$, then $C_{q}\left(J, u_{0}\right) \cong \delta_{q, l} \mathbb{F}$ for $l=\mu\left(u_{0}\right)$ or $l=\mu\left(u_{0}\right)+v\left(u_{0}\right)$.

Proposition 2.2 ([19-23]) Let 0 be an isolated critical point of $J \in C^{2}(E, \mathbb{R})$ with finite Morse index $\mu(0)$ and nullity $v(0)$. Assume that $J$ has a local linking at 0 with respect to a direct sum decomposition $E=E^{-} \oplus E^{+}, l=\operatorname{dim} E^{-}<\infty$, i.e., there exists $\rho>0$ such that

$$
\begin{aligned}
& J(z) \leq 0 \quad \text { for } z \in E^{-},\|u\| \leq \rho, \\
& J(u) \geq 0 \quad \text { for } u \in E^{+},\|u\| \leq \rho .
\end{aligned}
$$

Then $C_{q}(J, 0) \cong \delta_{q, l} \mathbb{\mathbb { F }}$ for either $l=\mu(0)$ or $l=\mu(0)+\nu(0)$.

Proposition 2.3 ([16,19]) Let E be a real Banach space with $E=X \oplus Y$ and suppose that $l=\operatorname{dim} X$ is finite. Assume that $J \in C^{2}(E, \mathbb{R})$ satisfies the $(P S)$ condition and

$\left(\mathrm{H}_{1}\right)$ there exist $\rho>0$ and $\alpha>0$ such that

$$
J(u)>\alpha, \quad u \in Y \cap B_{\rho},
$$

where $B_{\rho}=\{u \in E \mid\|u\| \leq \rho\}$,

$\left(\mathrm{H}_{2}\right)$ there exist $R>\rho>0$ and $\phi \in Y$ with $\|\phi\|=1$ such that

$$
J(u) \leq \alpha, \quad u \in \partial Q
$$

where $Q=\{u=v+s \phi \mid v \in X,\|v\| \leq R, 0 \leq s \leq R\}$.

Then $J$ has a critical point $u_{*}$ with $J\left(u_{*}\right)=c_{*} \geq \alpha$ and

$$
C_{l+1}\left(J, u_{*}\right) \nsucceq 0 .
$$

\section{Auxiliary results}

We first show that the functional $J$ satisfies the (PS) condition. 
Lemma 3.1 Assume that $f$ satisfies $\left(f_{\infty}^{+}\right)$or $\left(f_{\infty}^{-}\right)$, then J defined by (1.4) satisfies the (PS) condition.

Proof We only prove the case where $\left(f_{\infty}^{+}\right)$holds; the other case can be proved similarly. Let $\left\{u_{n}\right\} \subset E$ be such that

$$
\left|J\left(u_{n}\right)\right| \leq C, \quad n \in \mathbb{N}, \quad J^{\prime}\left(u_{n}\right) \rightarrow 0 \quad \text { as } n \rightarrow \infty .
$$

We only need to show that $\left\{u_{n}\right\}$ is bounded. Taking positive number $\alpha \in(1 / \theta, 1 / 2)$, it follows from (3.1) that there exists $K \in \mathbb{N}$ such that

$$
C+\alpha\left\|u_{n}\right\| \geq J\left(u_{n}\right)-\alpha\left\langle J^{\prime}\left(u_{n}\right), u_{n}\right\rangle, \quad n>K .
$$

By $\left(f_{\infty}^{+}\right)$, there exist $C_{1}, C_{2}>0$ such that

$$
\begin{aligned}
& F(k, x) \geq C_{1}\left(|x|^{\theta}-1\right), \quad x \in \mathbb{R}, k \in \mathbb{Z}[1, N], \\
& |\theta F(k, x)-f(k, x) x| \leq C_{2}, \quad|x| \leq r, k \in \mathbb{Z}[1, N] .
\end{aligned}
$$

Hence, by (1.2), $\left(f_{\infty}^{+}\right),(3.2)$ and (3.3), we have

$$
\begin{aligned}
C+\alpha\left\|u_{n}\right\| \geq & J\left(u_{n}\right)-\alpha\left\langle J^{\prime}\left(u_{n}\right), u_{n}\right\rangle \\
= & \left(\frac{1}{2}-\alpha\right)\left\langle A u_{n}, u_{n}\right\rangle-\left(\frac{1}{2}-\alpha\right) \lambda_{m}\left\|u_{n}\right\|^{2} \\
& -\sum_{k=1}^{N}\left[F\left(k, u_{n}(k)\right)-\alpha f\left(k, u_{n}(k)\right) u_{n}(k)\right] \\
\geq & \left(\alpha-\frac{1}{2}\right) \lambda_{m}\left\|u_{n}\right\|^{2}-\sum_{\left|u_{n}(k)\right|>r}\left[F\left(k, u_{n}(k)\right)-\alpha f\left(k, u_{n}(k)\right) u_{n}(k)\right] \\
& -\sum_{\left|u_{n}(k)\right| \leq r}\left[F\left(k, u_{n}(k)\right)-\alpha f\left(k, u_{n}(k)\right) u_{n}(k)\right] \\
\geq & \left(\alpha-\frac{1}{2}\right) \lambda_{m}\left\|u_{n}\right\|^{2}+(\theta \alpha-1) \sum_{k=1}^{N} F\left(k, u_{n}(k)\right) \\
& -\alpha \sum_{\left|u_{n}(k)\right| \leq r}\left[\theta F\left(k, u_{n}(k)\right)-f\left(k, u_{n}(k)\right) u_{n}(k)\right] \\
\geq & \left(\alpha-\frac{1}{2}\right) \lambda_{m}\left\|u_{n}\right\|^{2}+(\theta \alpha-1) C_{1}\left(\left\|u_{n}\right\|_{\theta}^{\theta}-N\right)-N C_{2} \\
\geq & \left(\alpha-\frac{1}{2}\right) \lambda_{m}\left\|u_{n}\right\|^{2}+(\theta \alpha-1) C_{1} a_{\theta}^{\theta}\left\|u_{n}\right\|^{\theta} \\
& -N\left[(\theta \alpha-1) C_{1}+C_{2}\right], \quad n>K .
\end{aligned}
$$

Since $\theta>2$ and $\alpha \in\left(\frac{1}{\theta}, \frac{1}{2}\right)$, we get that $\left\{u_{n}\right\}$ is bounded.

Remark 3.1 By the proof of Lemma 3.1, we have that if $f$ satisfies $\left(f_{\infty}^{+}\right)$or $\left(f_{\infty}^{-}\right)$, then $-J$ satisfies the (PS) condition. 
For $k \in \mathbb{Z}\left[0, N_{1}-1\right]$, defining

$$
E_{k}=\operatorname{span}\left\{\phi_{0}, \phi_{1}, \psi_{1}, \ldots, \phi_{k}, \psi_{k}\right\}, \quad E=E_{k} \oplus E_{k}^{\perp}, \quad \omega_{k}=\operatorname{dim} E_{k},
$$

then $\omega_{k}=2 k+1$ and

$$
E_{k}^{\perp}= \begin{cases}\operatorname{span}\left\{\phi_{k+1}, \psi_{k+1}, \ldots, \phi_{N_{1}}, \psi_{N_{1}}\right\} & \text { while } N \text { is odd } \\ \operatorname{span}\left\{\phi_{k+1}, \psi_{k+1}, \ldots, \phi_{N_{1}-1}, \psi_{N_{1}-1}, \phi_{N_{1}}\right\} & \text { while } N \text { is even }\end{cases}
$$

Now we construct a linking with respect to the decomposition $E=E_{m} \oplus E_{m}^{\perp}$ or $E=E_{m-1}^{\perp} \oplus$ $E_{m-1}$.

Lemma 3.2 Suppose that $f$ satisfies $\left(f_{0}\right)$ and $(f)$. Then

(i) for any fixed $m \in \mathbb{Z}\left[0, N_{1}-1\right]$, there exist $\rho_{m}>0$ and $\alpha_{m}>0$ such that

$$
J(u)>\alpha_{m} \quad \text { for } u \in E_{m}^{\perp} \text { with }\|u\|=\rho_{m} ;
$$

(ii) for any fixed $m \in \mathbb{Z}\left[1, N_{1}\right]$, there exist $\widetilde{\rho}_{m}>0$ and $\widetilde{\alpha}_{m}>0$ such that

$$
-J(u)>\widetilde{\alpha}_{m} \quad \text { for } u \in E_{m-1} \text { with }\|u\|=\widetilde{\rho}_{m} .
$$

Proof By $\left(f_{0}\right)$ and $(f)$, for any $\varepsilon>0$, there exists $C_{\varepsilon}>0$ such that

$$
|F(k, x)| \leq \frac{1}{2} \varepsilon x^{2}+C_{\varepsilon}|x|^{\tau}, \quad k \in \mathbb{Z}[1, N], x \in \mathbb{R} .
$$

(i) For any $u \in E_{m}^{\perp}$, it follows from (3.6) that

$$
\begin{aligned}
J(u) & =\frac{1}{2}\langle A u, u\rangle-\frac{1}{2} \lambda_{m}\|u\|^{2}-\sum_{k=1}^{N} F(k, u(k)) \\
& \geq \frac{1}{2}\left(\lambda_{m+1}-\lambda_{m}\right)\|u\|^{2}-\sum_{k=1}^{N}\left(\frac{1}{2} \varepsilon|u(k)|^{2}+C_{\varepsilon}|u(k)|^{\tau}\right) \\
& \geq \frac{1}{2}\left(\lambda_{m+1}-\lambda_{m}-\varepsilon\right)\|u\|^{2}-C_{\varepsilon} b_{\tau}^{\tau}\|u\|^{\tau} \\
& =\frac{1}{2}\left(\lambda_{m+1}-\lambda_{m}-\varepsilon\right)\|u\|^{2}-\widetilde{C}_{\varepsilon}\|u\|^{\tau} .
\end{aligned}
$$

Taking $\varepsilon=\frac{\lambda_{m+1}-\lambda_{m}}{2}$, we then have

$$
J(u) \geq \frac{1}{2} \varepsilon\|u\|^{2}-\widetilde{C}_{\varepsilon}\|u\|^{\tau}
$$

Because $\tau>2$, the function $h(\rho)=\frac{1}{2} \varepsilon \rho^{2}-\widetilde{C}_{\varepsilon} \rho^{\tau}$ defined on $[0, \infty)$ achieves its maximum

$$
h_{\max }=\frac{\tau-2}{2}\left(\tau^{-1} \varepsilon\right)^{\frac{\tau}{\tau-2}} \widetilde{C}_{\varepsilon}^{2-\tau}
$$




$$
\rho_{m}=\left(\varepsilon^{-1} \tau \widetilde{C}_{\varepsilon}\right)^{\frac{1}{2-\tau}}
$$

Hence we get (3.4), where $\alpha_{m}:=\frac{3}{4} h_{\max }$.

(ii) For any $u \in E_{m-1}$, by (3.6), we obtain

$$
\begin{aligned}
J(u) & =\frac{1}{2}\langle A u, u\rangle-\frac{1}{2} \lambda_{m}\|u\|^{2}-\sum_{k=1}^{N} F(k, u(k)) \\
& \leq \frac{1}{2}\left(\lambda_{m-1}-\lambda_{m}\right)\|u\|^{2}+\sum_{k=1}^{N}\left(\frac{1}{2} \varepsilon|u(k)|^{2}+C_{\varepsilon}|u(k)|^{\tau}\right) \\
& \leq \frac{1}{2}\left(\lambda_{m-1}-\lambda_{m}+\varepsilon\right)\|u\|^{2}+C_{\varepsilon} b_{\tau}^{\tau}\|u\|^{\tau} \\
& =\frac{1}{2}\left(\lambda_{m-1}-\lambda_{m}+\varepsilon\right)\|u\|^{2}+\widetilde{C}_{\varepsilon}\|u\|^{\tau} .
\end{aligned}
$$

Taking $\varepsilon=\frac{\lambda_{m}-\lambda_{m-1}}{2}$, we then obtain

$$
J(u) \leq-\frac{1}{2} \varepsilon\|u\|^{2}+\widetilde{C}_{\varepsilon}\|u\|^{\tau}
$$

As $\tau>2$, the function $h(\rho)=-\frac{1}{2} \varepsilon \rho^{2}+\hat{C}_{\varepsilon} \rho^{\tau}$ defined on $[0, \infty)$ achieves its minimum

$$
h_{\min }=-\frac{\tau-2}{2}\left(\tau^{-1} \varepsilon\right)^{\frac{\tau}{\tau-2}} \widetilde{C}_{\varepsilon}^{\frac{2}{2-\tau}}
$$

at

$$
\widetilde{\rho}_{m}=\left(\varepsilon^{-1} \tau \widetilde{C}_{\varepsilon}\right)^{\frac{1}{2-\tau}}
$$

Therefore, we obtain (3.5), where $\widetilde{\alpha}_{m}:=\frac{3}{4} h_{\min }$.

Now we define

$$
\begin{array}{ll}
B_{m}=\left\{u \in E_{m}^{\perp},\|u\|=\rho_{m}\right\}, & E^{m+1}=E_{m} \oplus \operatorname{span}\left\{\phi_{m+1}\right\}, \quad m \in \mathbb{Z}\left[0, N_{1}-1\right], \\
\widetilde{B}_{m}=\left\{u \in E_{m-1},\|u\|=\widetilde{\rho}_{m}\right\}, & \widetilde{E}^{m+1}=\operatorname{span}\left\{\phi_{m-1}\right\} \oplus E_{m-1}^{\perp}, \quad m \in \mathbb{Z}\left[1, N_{1}\right], \\
F^{-}(k, x)=\max \{-F(k, x), 0\}, & F^{+}(k, x)=\max \{F(k, x), 0\}, \\
M^{-}=\sup _{(k, x) \in \mathbb{Z}[1, N] \times \mathbb{R}} F^{-}(k, x), & M^{+}=\sup _{(k, x) \in \mathbb{Z}[1, N] \times \mathbb{R}} F^{+}(k, x) .
\end{array}
$$

Lemma 3.3 (i) Suppose that $f$ satisfies $\left(f_{\infty}^{+}\right)$. Then, for any fixed $m \in \mathbb{Z}\left[0, N_{1}-1\right]$, there exist $\sigma>0$ and $R>0$ such that when $M^{-} \leq \sigma$,

$$
J(u) \leq \alpha_{m} \quad \text { for } u \in \partial Q_{m},
$$

where $Q_{m}=\left\{u \in E^{m+1} \mid\|u\| \leq R, u=v_{m}+t \phi_{m+1}, v_{m} \in E_{m}, t \geq 0\right\}$. 
(ii) Suppose that $f$ satisfies $\left(f_{\infty}^{-}\right)$. Then, for any fixed $m \in \mathbb{Z}\left[1, N_{1}\right]$, there exist $\sigma>0$ and $R>0$ such that when $M^{+} \leq \sigma$,

$$
-J(u) \leq \widetilde{\alpha}_{m}, \quad \text { for } u \in \partial \widetilde{Q}_{m}
$$

where $\widetilde{Q}_{m}=\left\{u \in \widetilde{E}^{m+1} \mid\|u\| \leq R, u=t \phi_{m-1}+v_{m-1}, v_{m-1} \in E_{m-1}, t \geq 0\right\}$.

Proof We only prove (i); the proof of (ii) is similar. For any $u \in E^{m+1}$, it follows from $\left(f_{\infty}^{+}\right)$ and (3.2) that

$$
\begin{aligned}
J(u) & =\frac{1}{2}\langle A u, u\rangle-\frac{1}{2} \lambda_{m}\|u\|^{2}-\sum_{k=1}^{N} F(k, u(k)) \\
& \leq \frac{1}{2}\left(\lambda_{m+1}-\lambda_{m}\right)\|u\|^{2}-\sum_{k=1}^{N} C_{1}\left(|u(k)|^{\theta}-1\right) \\
& \leq \frac{1}{2}\left(\lambda_{m+1}-\lambda_{m}\right)\|u\|^{2}-C_{1} a_{\theta}^{\theta}\|u\|^{\theta}+N C_{1} .
\end{aligned}
$$

As $\theta>2$, we get

$$
J(u) \rightarrow-\infty, \quad u \in E^{m+1},\|u\| \rightarrow \infty .
$$

Therefore, there exists $R>\rho_{m}$ such that

$$
J(u) \leq 0, \quad u \in E^{m+1},\|u\|=R .
$$

For $u \in E_{m}$,

$$
\begin{aligned}
J(u) & =\frac{1}{2}\langle A u, u\rangle-\frac{1}{2} \lambda_{m}\|u\|^{2}-\sum_{k=1}^{N} F(k, u(k)) \\
& \leq-\sum_{F(k, u(k)) \leq 0} F(k, u(k)) \\
& \leq N M^{-} .
\end{aligned}
$$

Taking $\sigma=\frac{\alpha_{m}}{N}$, when $M^{-} \leq \sigma$,

$$
J(u) \leq \alpha_{m} \quad \text { for } u \in E_{m} \text { with }\|u\| \leq R .
$$

By (3.8) and (3.9), we get (3.7).

\section{Main results and proofs}

In this section, we give our main results and proofs. First, we compute the critical groups of $J$ at both infinity and the origin.

Lemma 4.1 Assume that $f$ satisfies $\left(f_{0}\right)$. Then

(i) $C_{q}(J, \infty) \cong \delta_{q, N} \mathbb{F}$ iff satisfies $\left(f_{\infty}^{+}\right)$.

(ii) $C_{q}(-J, \infty) \cong \delta_{q, N} \mathbb{F}$ iff satisfies $\left(f_{\infty}^{-}\right)$. 
Proof We only prove (i); (ii) can be proved similarly. Fix $\lambda_{m} \in \mathbb{R}$. Defining $B=\{u \in E \mid$ $\|u\| \leq 1\}$, we then have $\partial B=\{u \in E \mid\|u\|=1\}$. For any $u \in \partial B$ and $t>0$, it follows from (3.2) and (1.3) that

$$
\begin{aligned}
J(t u) & =\frac{1}{2} t^{2}\langle A u, u\rangle-\frac{1}{2} \lambda_{m} t^{2}-\sum_{k=1}^{N} F(k, t u(k)) \\
& \leq \frac{1}{2}\left(\lambda_{N_{1}}-\lambda_{m}\right) t^{2}-\sum_{k=1}^{N} C_{1}\left(|t u(k)|^{\theta}-1\right) \\
& \leq \frac{1}{2}\left(\lambda_{N_{1}}-\lambda_{m}\right) t^{2}-C_{1} a_{\theta}^{\theta} t^{\theta}+N C_{1} .
\end{aligned}
$$

As $\theta>2$, this implies that

$$
J(t u) \rightarrow-\infty \quad \text { as } t \rightarrow+\infty .
$$

On the other hand, by $\left(f_{\infty}^{+}\right)$, we have

$$
\begin{aligned}
\frac{d J(t u)}{d t} & =t\langle A u, u\rangle-\lambda_{m} t-\sum_{k=1}^{N} f(k, t u(k)) u(k) \\
& =\frac{1}{t}\left(2 J(t u)+\sum_{k=1}^{N}(2 F(k, t u(k))-f(k, t u(k)) t u(k))\right) \\
& \leq \frac{1}{t}\left(2 J(t u)+\sum_{|t u(k)| \leq r}(2 F(k, t u(k))-f(k, t u(k)) t u(k))\right) \\
& \leq \frac{1}{t}\left(2 J(t u)+N M_{1}\right),
\end{aligned}
$$

where $M_{1}:=\max _{\{|x| \leq r, k \in \mathbb{Z}[1, N]\}}|2 F(k, x)-f(k, x) x|$. Hence, for any fixed number $a$ with $2 a<$ $-N M_{1}$, we obtain that

$$
J(t u) \leq a \quad \text { implies } \quad \frac{d J(t u)}{d t}<0 .
$$

By $J(0)=0$, (4.1) and (4.2), we get that for any $u \in \partial B$, there exists a unique $\xi(u)>0$ such that

$$
J(\xi(u) u)=a, \quad u \in \partial B
$$

By (4.3) and the implicit function theorem, we obtain that $\xi \in C(\partial B, \mathbb{R})$. Define $h$ : $E \backslash\{0\} \rightarrow \mathbb{R}$ as

$$
h(u)= \begin{cases}1 & \text { if } J(u) \leq a \\ \|u\|^{-1} \xi\left(\|u\|^{-1} u\right) & \text { if } J(u)>a, u \neq 0\end{cases}
$$

Then $h \in C(E \backslash\{0\}, \mathbb{R})$. Now define $\eta:[0,1] \times E \backslash\{0\} \rightarrow E \backslash\{0\}$ as

$$
\eta(t, u)=(1-t) u+t h(u) u
$$


Clearly, $\eta$ is continuous, and for any $u \in E \backslash\{0\}$ with $J(u)>a$, it follows from (4.3) that

$$
J(\eta(1, u))=J\left(\xi\left(\|u\|^{-1} u\right)\|u\|^{-1} u\right)=a .
$$

Therefore

$$
\eta(1, u) \in J^{a}, \quad \forall u \in E \backslash\{0\}, \quad \eta(t, u)=u, \quad \forall t \in[0,1], u \in J^{a} .
$$

So $J^{a}$ is a strong deformation retract of $E \backslash\{0\}$. Notice that $\operatorname{dim} E=N$. Hence we have

$$
C_{q}(J, \infty):=H_{q}\left(E, J^{a}\right) \cong H_{q}(E, E \backslash\{0\}) \cong H(B, \partial B) \cong \delta_{q, N} \mathbb{F} .
$$

Lemma 4.2 Assume that $f$ satisfies $\left(f_{0}\right)$.

(i) Iff satisfies $\left(f_{0}^{-}\right)$, then for $m \in \mathbb{Z}\left[1, N_{1}\right]$,

$$
C_{q}(J, 0) \cong \delta_{q, 2 m-1} \mathbb{F}, \quad C_{q}(-J, 0) \cong \delta_{q, N-2 m+1} \mathbb{F} .
$$

(ii) Iff satisfies $\left(f_{0}^{+}\right)$, then for $m \in \mathbb{Z}\left[0, N_{1}-1\right]$,

$$
C_{q}(J, 0) \cong \delta_{q, 2 m+1} \mathbb{F}, \quad C_{q}(-J, 0) \cong \delta_{q, N-2 m-1} \mathbb{F} .
$$

Proof By $\left(f_{0}\right)$ and (1.6), we have $J^{\prime \prime}(0)=A-\lambda_{m} I_{N}$.

It follows from $\left(f_{0}\right)$ that $u=0$ is a degenerate critical point of $J$ with Morse index $\mu(0)=$ $\omega_{m-1}$ and nullity $v(0)$. This implies that $u=0$ is a degenerate critical point of $-J$ with Morse index $\widetilde{\mu}(0)=N-\omega_{m}$ and nullity $\widetilde{v}(0)=v(0)$.

(i) By $\left(f_{0}^{-}\right)$, we can verify that $J$ has a local linking structure at 0 with respect to $E=$ $E_{m-1} \oplus E_{m-1}^{\perp}$ (see [12]). That implies $-J$ has a local linking structure at 0 with respect to $E=E_{m-1}^{\perp} \oplus E_{m-1}$. Notice that $\omega_{m-1}=2 m-1$ and $\widetilde{\mu}(0)+\widetilde{\nu}(0)=N-2 m+1$ for $m \in \mathbb{Z}\left[1, N_{1}\right]$. Using Proposition 2.2, we get

$$
C_{q}(J, 0) \cong \delta_{q, 2 m-1} \mathbb{F}, \quad C_{q}(-J, 0) \cong \delta_{q, N-2 m+1} \mathbb{F} .
$$

(ii) Similarly, by $\left(f_{0}^{+}\right)$, we can verify that $J$ has a local linking structure at 0 with respect to $E=E_{m} \oplus E_{m}^{\perp}$ (see [12]). That implies $-J$ has a local linking structure at 0 with respect to $E=E_{m}^{\perp} \oplus E_{m}$. Notice that $\mu(0)+v(0)=\omega_{m}=2 m+1$ for $m \in \mathbb{Z}\left[0, N_{1}-1\right]$. By Proposition 2.2, we have

$$
C_{q}(J, 0) \cong \delta_{q, 2 m+1} \mathbb{F}, \quad C_{q}(-J, 0) \cong \delta_{q, N-2 m-1} \mathbb{F} .
$$

Next, we give our main results.

Theorem 4.1 Letf satisfy $\left(f_{0}\right)$ and $(f)$.

(i) Iff satisfies $\left(f_{\infty}^{+}\right)$, then for any fixed $m \in \mathbb{Z}\left[0, N_{1}-1\right]$, there exists $\sigma>0$ such that when $M^{-} \leq \sigma,(P)$ has at least one nonzero periodic solution $u_{1}$ satisfying

$$
C_{2 m+2}\left(J, u_{1}\right) \varsubsetneqq 0 .
$$


(ii) If $f$ satisfies $\left(f_{\infty}^{-}\right)$, then for any fixed $m \in \mathbb{Z}\left[1, N_{1}\right]$, there exists $\sigma>0$ such that when $M^{+} \leq \sigma,(P)$ has at least one nonzero periodic solution $u_{1}$ satisfying

$$
C_{N-2 m+2}\left(-J, u_{1}\right) \supsetneqq 0 \text {. }
$$

Theorem 4.2 Let $f$ satisfy $\left(f_{0}\right)$ and $(f)$.

(i) Iff satisfies $\left(f_{0}^{-}\right)$and $\left(f_{\infty}^{+}\right)$, then, for any fixed $m \in \mathbb{Z}\left[1, N_{1}-2\right]$, there exists $\sigma>0$ such that when $M^{-} \leq \sigma,(P)$ has at least three nonzero periodic solutions.

(ii) Iff satisfies $\left(f_{0}^{+}\right)$and $\left(f_{\infty}^{-}\right)$, then, for any fixed $m \in \mathbb{Z}\left[2, N_{1}-1\right]$, there exists $\sigma>0$ such that when $M^{+} \leq \sigma,(P)$ has at least three nonzero periodic solutions.

Finally, we prove our main results.

Proof of Theorem 4.1 We only prove (i); the proof of (ii) is similar. It follows from $\left(f_{\infty}^{+}\right)$ and Lemma 3.1 that $J$ satisfies the (PS) condition. By Lemmas 3.2 and 3.3, $J$ satisfies $\left(\mathrm{H}_{1}\right)$ and $\left(\mathrm{H}_{2}\right)$. This implies that $B_{m}$ and $\partial Q_{m}$ homologically link with respect to the direct sum decomposition $E=E_{m} \oplus E_{m}^{\perp}$ (see Example 3 of Chapter II in [16]). Notice that $\operatorname{dim} E_{m}=$ $2 m+1$. Applying Proposition 2.3, we get that $J$ has a critical point $u_{1}$ such that $J\left(u_{1}\right)=c_{1} \geq$ $\alpha_{m}$ and (4.4). Moreover, it follows from $\left(f_{0}\right)$ that $J(0)=0$. Hence $u_{1} \neq 0$.

Proof of Theorem 4.2 We only prove (i); (ii) can be proved similarly. It follows from Lemma 4.1(i) that

$$
C_{q}(J, \infty) \cong \delta_{q, N} \mathbb{F}
$$

Using Lemma 4.2(i), we have

$$
C_{q}(J, 0) \cong \delta_{q, 2 m-1} \mathbb{F} \text {. }
$$

By Theorem 4.1, we know that there exists $\sigma>0$ such that when $M^{-} \leq \sigma,(P)$ has at least one nonzero periodic solution $u_{1}$ satisfying (4.4).

By Proposition 2.1(i), we have

$$
C_{q}\left(J, u_{1}\right) \cong 0 \quad \text { for } q \notin\left[\mu\left(u_{1}\right), \mu\left(u_{1}\right)+v\left(u_{1}\right)\right]
$$

Combining with (4.4), we get that $2 m+2 \in\left[\mu\left(u_{1}\right), \mu\left(u_{1}\right)+v\left(u_{1}\right)\right]$. Note that $v\left(u_{1}\right)=$ $\operatorname{dim} \operatorname{ker} J^{\prime \prime}\left(u_{1}\right) \leq 2$.

(1) If $v\left(u_{1}\right)=0$, then by Proposition 2.1(ii) and (4.4),

$$
C_{q}\left(J, u_{1}\right) \cong \delta_{q, 2 m+2} \mathbb{F} \text {. }
$$

(2) If $v\left(u_{1}\right)=1$, then $2 m+2=\mu\left(u_{1}\right)$ or $2 m+2=\mu\left(u_{1}\right)+v\left(u_{1}\right)$. By Proposition 2.1(iii),

$$
C_{q}\left(J, u_{1}\right) \cong \delta_{q, 2 m+2} \mathbb{F} \text {. }
$$


(3) If $v\left(u_{1}\right)=2$, then $2 m+2=\mu\left(u_{1}\right)$ or $2 m+2=\mu\left(u_{1}\right)+v\left(u_{1}\right)$ or $C_{q}\left(J, u_{1}\right) \cong 0$ for $q \neq$ $\mu\left(u_{1}\right)+1$. It follows from Proposition 2.1(iii) and (4.4) that

$$
C_{q}\left(J, u_{1}\right) \cong \begin{cases}\delta_{q, 2 m+2} \mathbb{F} & \text { if } 2 m+2=\mu\left(u_{1}\right) \text { or } 2 m+2=\mu\left(u_{1}\right)+v\left(u_{1}\right) \\ 0 & \forall q \neq 2 m+2\end{cases}
$$

Thus, we conclude that

$$
C_{q}\left(J, u_{1}\right) \cong \begin{cases}\delta_{q, 2 m+2} \mathbb{F} & \text { if } v\left(u_{1}\right)=0,1 \\ 0 & \forall q \neq 2 m+2, \text { if } v\left(u_{1}\right)=2\end{cases}
$$

Assume that $\mathcal{K}=\left\{0, u_{1}\right\}$. Then the $2 m$ th Morse inequality (2.1) is expressed as $-1>0$. This is impossible. Thus $J$ must have another nonzero critical point $u_{2}$. By Morse theory, we have either

$$
C_{2 m}\left(J, u_{2}\right) \nRightarrow 0
$$

or

$$
C_{2 m-2}\left(J, u_{2}\right) \varsubsetneqq 0 \text {. }
$$

Suppose that (4.9) holds. Then it follows from (4.9) and Proposition 2.1(i), (iii) that

$$
C_{q}\left(J, u_{2}\right) \cong \begin{cases}\delta_{q, 2 m} \mathbb{F} & \text { if } v\left(u_{2}\right)=0,1 \\ 0 & \forall q \neq 2 m, \text { if } v\left(u_{2}\right)=2\end{cases}
$$

Noticing that $m \in \mathbb{Z}\left[1, N_{1}-2\right]$, we have that

$$
\begin{cases}2 m+3 \leq N & \text { while } N \text { is odd } \\ 2 m+3<N & \text { while } N \text { is even }\end{cases}
$$

Assume that $\mathcal{K}=\left\{0, u_{1}, u_{2}\right\}$. We will divide the consideration into four cases.

(1) $v\left(u_{1}\right)=0,1$ and $v\left(u_{2}\right)=0,1$. By (4.6)-(4.8), (4.11) and (4.12), we get

$$
\begin{cases}(2.2) \text { is described as } 1=-1 & \text { while } N \text { is odd, } \\ \text { the } 2 m+3 \text { th }(2.1) \text { is expressed as }-1 \geq 0 & \text { while } N \text { is even. }\end{cases}
$$

These are impossible.

(2) $v\left(u_{1}\right)=2$ and $v\left(u_{2}\right)=2$. By the $2 m$ th and $2 m+1$ th Morse inequalities, we get that

$$
\operatorname{rank} C_{2 m}\left(J, u_{2}\right)=1
$$

It then follows from (4.6)-(4.8), (4.11), (4.12) and (4.14) that

$$
\begin{cases}(2.2) \text { is expressed as } \operatorname{rank} C_{2 m+2}\left(J, u_{1}\right)=-1 & \text { while } N \text { is odd, } \\ \text { the } 2 m+3 \text { th }(2.1) \text { is expressed as }-\operatorname{rank} C_{2 m+2}\left(J, u_{1}\right) \geq 0 & \text { while } N \text { is even }\end{cases}
$$

These contradict (4.8). 
(3) $v\left(u_{1}\right)=0,1$ and $v\left(u_{2}\right)=2$. We have (4.14) and (4.13). These are impossible.

(4) $v\left(u_{1}\right)=2$ and $v\left(u_{2}\right)=0,1$. Then we have (4.15), which contradicts (4.8).

Hence, $J$ must have a third nonzero critical point $u_{3}$. The proof of the case where (4.10) holds is similar. This completes the proof.

\section{Competing interests}

The authors declare that they have no competing interests.

\section{Authors' contributions}

The authors have made this manuscript independently. The authors read and approved the final version.

\section{Acknowledgements}

The authors thank the reviewers for their valuable comments and suggestions. In addition, this work is partly supported by the National Natural Science Foundation of China (No. 51278325) and by the Natural Science Foundations of Shanxi (Nos. $2011011002-4,2012011004-3$ )

Received: 15 November 2013 Accepted: 12 August 2014 Published: 09 Sep 2014

\section{References}

1. Ma, RY, Ma, HL: Unbounded perturbations of nonlinear discrete periodic problem at resonance. Nonlinear Anal. 70 2602-2613 (2009)

2. Guo, ZM, Yu, JS: The existence of periodic and subharmonic solutions for second-order superlinear difference equations. Sci. China Ser. A 33, 226-235 (2003) (in Chinese)

3. Agarwal, RP, Perera, K, O'Regan, D: Multiple periodic solutions of singular and nonsingular discrete problems via variational methods. Nonlinear Anal. TMA 58, 69-73 (2004)

4. Agarwal, RP, Perera, K, O'Regan, D: Multiple positive solutions of singular discrete $p$-Laplacian problems via variational methods. Adv. Differ. Equ. 2, 93-99 (2005)

5. Anderson, DR, Rachünková, I, Tisdell, CC: Solvability of discrete Neumann boundary value problems. J. Math. Anal. Appl. 331, 736-741 (2007)

6. Bonanno, G, Candito, P: Nonlinear difference equations investigated via critical point methods. Nonlinear Anal. 70, 3180-3186 (2009)

7. Candito, P, Giovannelli, N: Multiple solutions for a discrete boundary value problem involving the $p$-Laplacian Comput. Math. Appl. 56, 959-964 (2008)

8. Wang, D, Guan, W: Three positive solutions of boundary value problems for $p$-Laplacian difference equations. Comput. Math. Appl. 55, 1943-1949 (2008)

9. Li, Y, Lu, L: Existence of positive solutions of $p$-Laplacian difference equations. Appl. Math. Lett. 19, 1019-1023 (2006)

10. Zhu, BS, Yu, JS: Multiple positive solutions for resonant difference equations. Math. Comput. Model. 49, 1928-1936 (2009)

11. Zheng, B, Xiao, HF: Existence of multiple solutions of a second-order difference boundary value problem. Int. J. Math. Math. Sci. 2010, Article ID 907453 (2010)

12. Liu, JS, Wang, SL, Zhang, JM: Multiple solutions for boundary value problems of second-order difference equations with resonance. J. Math. Anal. Appl. 374, 187-196 (2011)

13. Wang, SL, Liu, JS, Zhang, JM, Zhang, FW: Existence of non-trivial solutions for resonant difference equations. J. Differ. Equ. Appl. 19, 209-222 (2013)

14. Wang, SL, Liu, JS: Nontrivial solutions of a second order difference systems with multiple resonance. Appl. Math. Comput. 218, 9342-9352 (2012)

15. Zhang, XS, Wang, D: Multiple periodic solutions for difference equations with double resonance at infinity. Adv. Differ Equ. 2011, Article ID 15 (2011)

16. Chang, KC: Infinite Dimensional Morse Theory and Multiple Solutions Problems. Birkhäuser, Boston (1993)

17. Mawhin, J, Willem, M: Critical Point Theory and Hamiltonian Systems. Springer, Berlin (1989)

18. Bartsch, T, Li, SJ: Critical point theory for asymptotically quadratic functional and application to the problems with resonance. Nonlinear Anal. 28, 419-441 (1997)

19. Liu, JQ: A Morse index for a saddle point. Syst. Sci. Math. Sci. 2, 32-39 (1989)

20. Su, JB: Semilinear elliptic boundary value problems with double resonance between two consecutive eigenvalues. Nonlinear Anal. 48, 881-895 (2002)

21. Perera, K: Critical groups of critical points produced by local linking with applications. Abstr. Appl. Anal. 3, 437-446 (1998)

22. Brezis, H, Nirenberg, L: Remarks on finding critical points. Commun. Pure Appl. Math. 64, 939-963 (1991)

23. Li, SJ, Willem, M: Applications of local linking to critical point theory. J. Math. Anal. Appl. 189, 6-32 (1995) 